\title{
Free Standing Optics for Hot Operation in the Water Window and EUV
}

\section{$\underline{\text { P. Charalambous }}^{1}$}

1. ZONEPLATES.COM, 8 South Way (FF), Claverings Industrial Estate, London N9 0AB, UK Email:pambos@ZonePlates.com

The motivation for designing the new $\mathrm{SiN}$ range, was the need for optics that could image samples heated to a few hundred degrees only a few mm away (focal length, Water Window). In this set up, conventional Gold or Nickel on SiN ZPs would typically last only hours before de-lamination and ultimate destruction! As expected, our Tungsten on SiN ZPs performed much better, because of the close thermal matching between the Tungsten absorber and the SiN substrates. However, it soon became clear that a completely monolithic optic would be even better, because it would totally avoid bimetallic distortions and stresses on the optic, caused by the difference in the Linear Thermal Expansion Coefficients of the constituent materials[1]. Free standing ZPs for focussing Thermal Helium Atoms have been reported in the past [2], however focussing X-rays in hot environments, requires different design criteria regarding film thickness and composition. Our first generation Free Standing ZPs are made of SiN films, typically used as ZP substrates. The films used are LOW STRESS (or Silicon Rich) rather than stoichiometric, with a composition closer to $\mathrm{Si}_{4} \mathrm{~N}_{3}$ than $\mathrm{Si}_{3} \mathrm{~N}_{4}$ ! The film must be of sufficient thickness to permit the etching of the grooves of the optic to sufficient depth in order to obtain the required Diffraction Efficiency (DE).

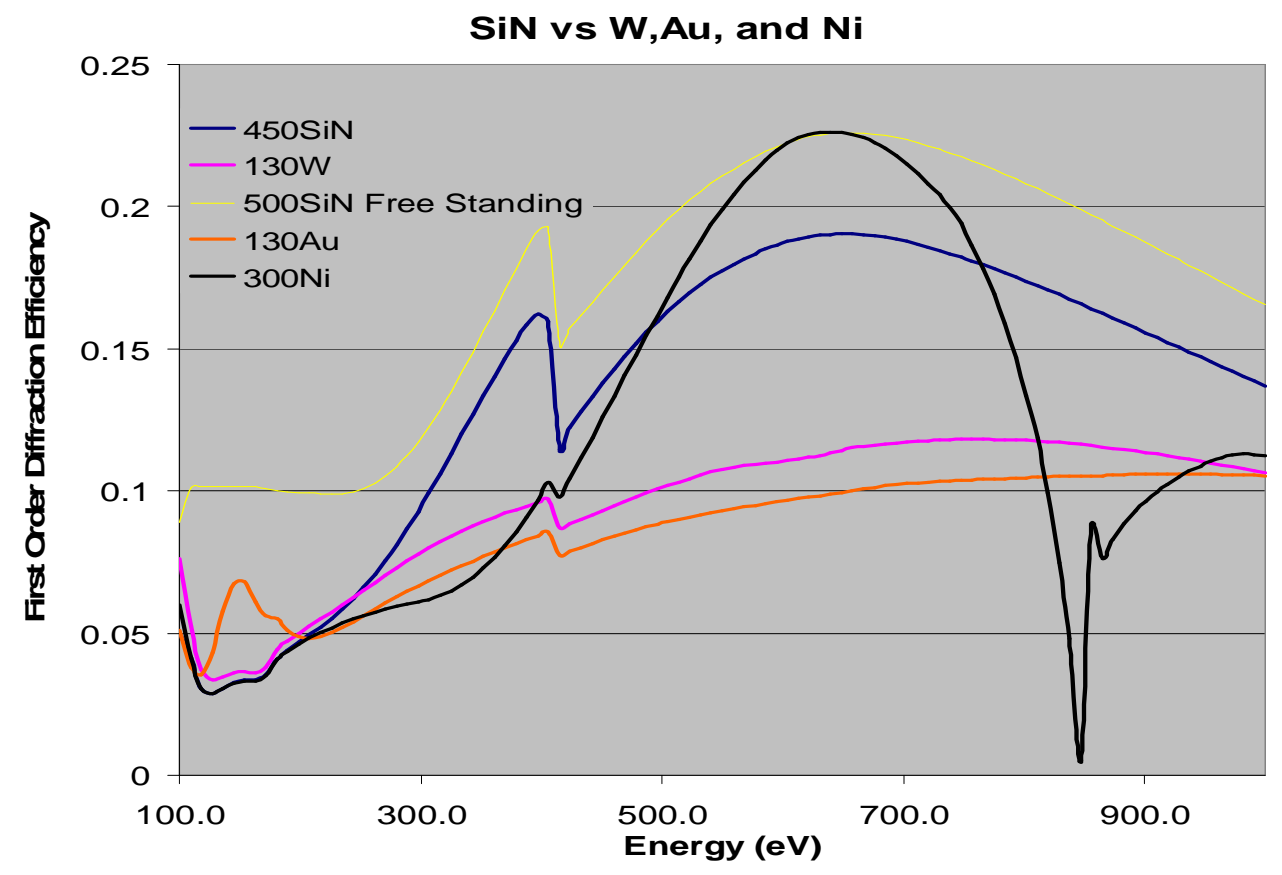

Fig 1. Comparison of the Diffraction Efficiency of optics in different materials and thickness (nm) optimized for operation in the Water Window. W, Au and Ni on 100nm SiN substrates.

In practice, we use $500 \mathrm{~nm}$ thick $\mathrm{SiN}$, and etch to a depth of 400-450 nm, leaving a residual 50-100 nm $\mathrm{SiN}$ film as a "substrate", which is perfectly acceptable for operation throughout the entire Water 
Window region and beyond. However, for EUV operation, the absorption and loss of intensity in the residual (substrate) film is still problematic! In the last year, we have been able to create a number of Free Standing optics by etching through the SiN film completely, thus eliminating the "substrate". Both versions, with residual "substrate", or free standing, fulfil the criteria for hot operation since they are entirely monolithic! In addition, referring to the graph of fig.1, it is clear that a free standing, $500 \mathrm{~nm}$ thick SiN ZP yields the best DE throughout the Water Window range, especially below $\sim 300 \mathrm{eV}$. The micrographs of fig. 2 show the inner and outer regions of one of our free standing SiN ZPs.
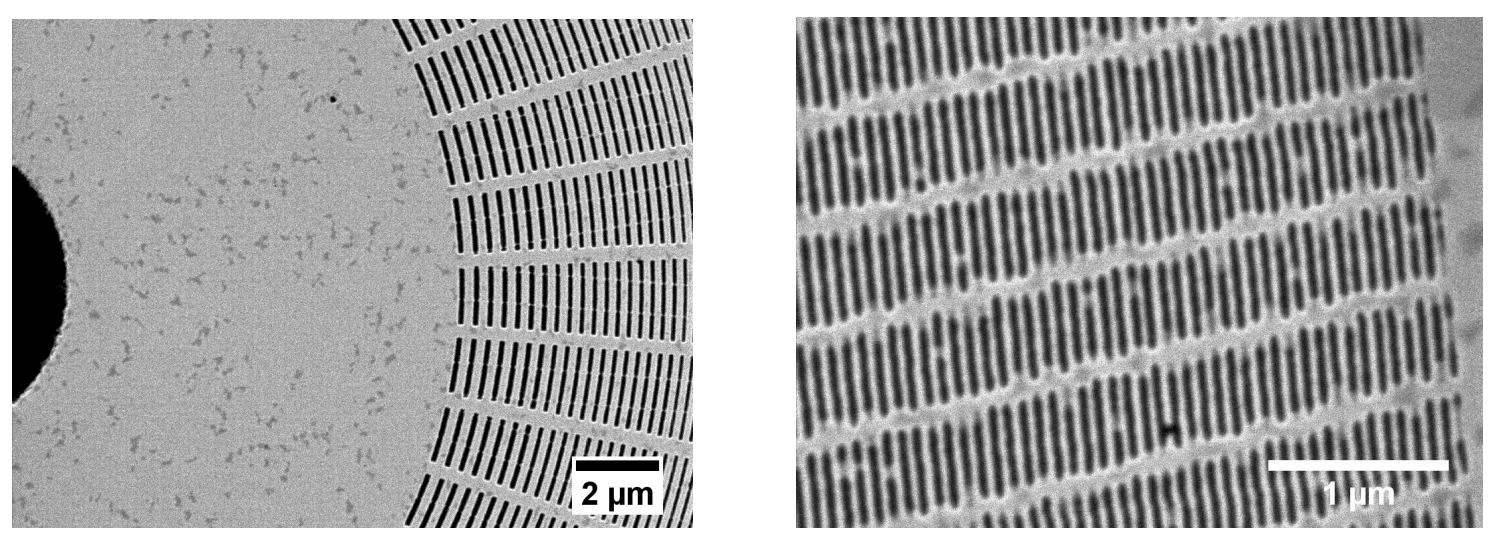

Fig. 2. Free Standing ZP in $500 \mathrm{~nm}$ SiN. Diameter $=160 \mu \mathrm{m}$, drn=40 nm

At present we are trying to create optics for operation at higher energies, namely $30 \mathrm{KeV}$ and beyond, but at these energies, the thickness of the absorber has to be increased dramatically! We have been able to deposit Tungsten films up to a thickness of $3 \mu \mathrm{m}$ for a number of years, and these have been successful, and reported in the past [3]. However, for reasonable DEs at these higher energies, we need a thickness of 5 or even $10 \mu \mathrm{m}$, More over, if these structures are free standing, they could be used with low photon energy/high brightness FELs, where heat dispersion is vital! However, increasing the thickness of our deposited films is difficult due to deposition stress!

Because of this, we are currently investigating a new approach. Starting with SOLID metal discs, a bore hole is created in the centre, thus creating a "window" where the metal is only $10 \mu \mathrm{m}$ thick or less. Next, the various mask layers are deposited on the flat side of the disc, and lithography and nano-structuring is performed as usual, finishing with Free Standing structures if necessary (for FEL operation). In addition to Tungsten, we are also investigating Tantalum and Molybdenum. Free standing Silicon ZPs for "Hot" operation have been reported in 2008 [4], however, since then, the power of FELs has increased dramatically, leading to much higher heat loading of the optics, and this is the motivation for attempting to move to refractory metals, which have much higher meting temperatures than Silicon.

[1]P. Charalambous, "Status of Nanofabrication at ZonePlates.com" Conference Proceedings XRM2005, pp. 127-129

[2]S. Rehbein et al., "Zone Plates for Helium Atom Focussing" Microelectronic Engineering 53, 685688 (2000)

[3]P. Charalambous, "Developments in the Fabrication of Zone Plates and other Nanostructures", Conference Proceedings XRM2010

[4]J. Vila-Comamala et al., "Silicon Fresnel zone plates for high heat load X-ray Microscopy", Microelectronic Engineering 85, 1241 (2008) 